\title{
Dynamic behaviors for inertial neural networks with reaction-diffusion terms and distributed delays
}

\section{Famei Zheng ${ }^{1 *}$}

"Correspondence:

hystzfm@163.com

${ }^{1}$ School of Mathematics and

Statistics, Huaiyin Normal University,

Huaian, Jiangsu, 223300, P.R. China

\section{勿 Springer}

\begin{abstract}
A class of inertial neural networks (INNs) with reaction-diffusion terms and distributed delays is studied. The existence and uniqueness of the equilibrium point for the considered system is obtained by topological degree theory, and a sufficient condition is given to guarantee global exponential stability of the equilibrium point. Finally, an example is given to show the effectiveness of the results in this paper.
\end{abstract}

Keywords: Inertial neural networks; Reaction-diffusion terms; Stability

\section{Introduction}

In 1997, Wheeler and Schieve [1] introduced inductance into neural networks and obtained a second-order neural network which is called inertial neural networks (INNs). In standard neural systems, inertial terms are introduced for biological backgrounds. Angelaki and Correia [2] studied a model of membrane resonance in pigeon semicircular canal type II hair cells which introduced inertial terms. The authors considered a quasi-active membrane behavior of neurons which can be modeled by introducing inductance. Inertial neural networks (INNs) are represented by second-order differential system. In INNs, inertial terms are described by the first-order derivative terms which have important meaning in biology, engineering technology, and information system, for more details, see e.g. [3-6]. Due to the inertial terms, it is very difficult to study the dynamic properties of the network system. Over the past years, many researchers have used different methods and techniques to study INNs in depth and obtained a large number of results. Tu, Cao, and Hayat [7] investigated the global dissipativity for INNs with time-varying delays and parameter uncertainties by using the generalized Halanay inequality, the matrix measure, and the matrix-norm inequality. Wang and Jiang [8] considered a class of impulsive INNs with time-varying delays. The global exponential stability in the Lagrange sense for INNs with delays was discussed in [4, 5]. Draye, Winters, and Cheron [9] studied a class of selfselected modular recurrent neural networks with postural and inertial subnetworks.

On the other hand, neural networks must be affected by reaction-diffusion terms, when neurons are moving in asymmetric neural networks. Hence, considering the activations change in space as well as in time is necessary for the research of neural networks. He

(c) The Author(s) 2021. This article is licensed under a Creative Commons Attribution 4.0 International License, which permits use sharing, adaptation, distribution and reproduction in any medium or format, as long as you give appropriate credit to the original author(s) and the source, provide a link to the Creative Commons licence, and indicate if changes were made. The images or other third party material in this article are included in the article's Creative Commons licence, unless indicated otherwise in a credit line to the material. If material is not included in the article's Creative Commons licence and your intended use is not permitted by statutory regulation or exceeds the permitted use, you will need to obtain permission directly from the copyright holder. To view a copy of this licence, visit http://creativecommons.org/licenses/by/4.0/. 
and Kang [10] studied a class of parabolic systems by using a semi-discrete method and a Lyapunov functional method. They obtained existence and several criteria of stability of the global generalized solutions for the considered systems. Li and Cao [11] investigated stochastic Cohen-Grossberg neural networks with time-varying delays and reaction-diffusion terms by using the Halanay inequality technique and the Lyapunov method. The criteria of delay-independent exponential stability were established for the above system. Carpenter [12] studied a singular perturbation problem with application to nerve impulse equations by the use of a geometric approach. In [13], the authors studied the exponential periodicity and stability of neural networks with Lipschitz continuous activation functions. The considered system in [13] contains reaction-diffusion terms and mixed delays.

Motivated by the above discussions, in this paper we are devoted to studying the existence and global asymptotic stability of the equilibrium point for a class reaction-diffusion neural networks with distributed delays subject to Dirichlet boundary conditions. By using the Lyapunov functional method, some new stability criteria are obtained which also guarantee the network will be asymptotically convergent to the equilibrium point. The theoretical methods developed in this paper have universal significance and can be easily extended to investigate many other types of neural networks with distributed delays. Our main contributions are summarized as follows.

(i) In this paper, we firstly study a new class of INNs which contains reaction-diffusion terms and distributed delays. In generally, the diffusion effect cannot be avoided in the neural networks. So we must consider that the space is varying with the time.

(ii) Due to influence of reaction-diffusion terms and distributed delays, we cannot easily obtain global exponential stability. By using the innovative mathematical analysis skills, we overcome the above difficulty.

(iii) It is nontrivial to establish a unified framework to handle reaction-diffusion terms and distributed time delays. Our method provides a useful reference for studying more complex systems.

Throughout this paper, let $S=\{1,2, \ldots, n\}$. For any $f=\left(f_{1}, \ldots, f_{n}\right) \in \mathbb{R}^{n}$, denote the norm $\|f\|_{1}=\sum_{k=1}^{n}|f|_{k}$. For any $u(t, x)=\left(u_{1}(t, x), \ldots, u_{n}(t, x)\right) \in \mathbb{R}^{n}$, define $\left\|u_{i}(t, x)\right\|_{2}=$ $\left(\int_{\Omega}\left|u_{i}(t, x)\right|^{2} d x\right)^{\frac{1}{2}}, i \in S$.

Lemma 1.1 ([14]) Let $H(\lambda, x):[0,1] \times \bar{\Omega} \rightarrow \mathbb{R}^{n}$ be a continuous homotopic mapping. If $H(\lambda, x)=y$ has no solutions in $\partial \Omega$ for $\lambda \in[0,1]$ and $y \in \mathbb{R}^{n} \backslash H(\lambda, \partial \Omega)$, where $\partial \Omega$ denotes the boundary of $\Omega$, then the topological degree $\operatorname{deg}(H(\lambda, x), \Omega, y)$ of $H(\lambda, x)$ is a constant which is independent of $\lambda$. Thus, $\operatorname{deg}(H(0, x), \Omega, y)=\operatorname{deg}(H(1, x), \Omega, y)$.

Lemma 1.2 ([14]) Let $H(x): \bar{\Omega} \rightarrow \mathbb{R}^{n}$ be a continuous mapping. If $\operatorname{deg}(H(x), \Omega, y) \neq 0$, then there exists at least one solution $H(x)=y$ in $\Omega$.

The rest of this paper is organized as follows. In Sect. 2, the reaction-diffusion neural networks with distributed delays are presented. In Sect. 3, the existence and global exponential stability of the equilibrium point for the considered model are studied. An example is presented to illustrate our theoretical results in Sect. 4. Finally, conclusions are drawn in Sect. 5. 


\section{Problem formulation}

The classic INNs with distributed delays are described as follows:

$$
\begin{aligned}
u_{i}^{\prime \prime}(t)= & -a_{i}(t) u_{i}^{\prime}(t)-b_{i}(t) u_{i}(t)+\sum_{j=1}^{n} p_{i j}(t) f_{j}\left(u_{j}(t)\right) \\
& +\sum_{j=1}^{n} \int_{-\infty}^{t} \sigma_{i j}(t-s) f_{j}\left(u_{j}(s)\right) d s+I_{i}(t),
\end{aligned}
$$

which has been extensively studied, see e.g. [4-6] and related references. By the use of the variable transformation function

$$
v_{i}(t)=\frac{d u_{i}(t)}{d t}+a_{i} u_{i}(t)
$$

system (2.1) can be rewritten as follows:

$$
\left\{\begin{aligned}
\frac{d u_{i}(t)}{d t}= & -a_{i} u_{i}(t)+v_{i}(t), \\
\frac{d v_{i}(t)}{d t}= & -b_{i} u_{i}(t)+\sum_{j=1}^{n} p_{i j} f_{j}\left(u_{j}(t)\right) \\
& +\sum_{j=1}^{n} q_{i j} \int_{-\infty}^{t} \sigma_{i j}(t-s) f_{j}\left(u_{j}(s)\right) d s+I_{i} .
\end{aligned}\right.
$$

Add the reaction-diffusion terms in (2.2), then

$$
\left\{\begin{aligned}
\frac{\partial u_{i}(t, x)}{\partial t}= & \sum_{k=1}^{l} \frac{\partial}{\partial x_{k}}\left(D_{i k} \frac{\partial u_{i}}{\partial x_{k}}\right)-a_{i} u_{i}+v_{i}(t, x), \\
\frac{\partial v_{i}(t, x)}{\partial t}= & \sum_{k=1}^{l} \frac{\partial}{\partial x_{k}}\left(D_{i k} \frac{\partial v_{i}}{\partial x_{k}}\right)-b_{i} u_{i}+\sum_{j=1}^{n} p_{i j} f_{j}\left(u_{j}\right) \\
& +\sum_{j=1}^{n} q_{i j} \int_{-\infty}^{t} \sigma_{i j}(t-s) f_{j}\left(u_{j}(s, x)\right) d s+I_{i},
\end{aligned}\right.
$$

where $i \in S, x=\left(x_{1}, \ldots, x_{l}\right)^{\top} \in \Xi \subset \mathbb{R}^{l}, \Xi$ is a bounded compact set and has smooth boundary, $u_{i}(t, x)$ denotes the state of $i$ th neuron at time $t$ and in space $x, I_{i}$ is the external input on the $i$ th neurons, $f_{j}(\cdot)$ is the activation function which is a continuous function, $\sigma_{i j}:[0, \infty) \rightarrow[0, \infty)$ is a delay kernel, $a_{i}, b_{i}, D_{i k}, p_{i j}$, and $q_{i j}$ are constants, $a_{i}>0$ is the damping coefficient, $b_{i}>0$ denotes the strength of different neuron, $D_{i k}=D_{i k}(t, x, u) \geq 0$ is the transmission diffusion operator, $p_{i j}$ and $q_{i j}$ are connection weights. The boundary conditions and initial conditions of system (2.3) are given by

$$
\begin{cases}\frac{\partial u_{i}}{\partial n}=\left(\frac{\partial u_{i}}{\partial x_{1}}, \ldots, \frac{\partial u_{i}}{\partial x_{l}}\right)^{\top}=0, & i \in S \\ \frac{\partial v_{i}}{\partial n}=\left(\frac{\partial v_{i}}{\partial x_{1}}, \ldots, \frac{\partial v_{i}}{\partial x_{l}}\right)^{\top}=0, & i \in S\end{cases}
$$

and

$$
\begin{cases}u_{i}(s, x)=\phi_{i}(s, x), & s \in(-\infty, 0], i \in S, \\ v_{i}(s, x)=\psi_{i}(s, x), & s \in(-\infty, 0], i \in S .\end{cases}
$$

Remark 2.1 When reaction-diffusion terms $D_{i k}=0, u_{i}(t, x)=u_{i}(t)$, and $v_{i}(t, x)=v_{i}(t)$, system (2.3) is changed into system (2.2). System (2.2) and system (2.3) are different types of equations. Hence system (2.3) is not equivalent to original system (2.2). This paper aims to 
study a class of inertial neural networks (2.3) with reaction-diffusion terms and distributed delays.

\section{Main results}

We need the following assumptions for activation functions and delay kernels functions:

$\left(\mathrm{H}_{1}\right)$ There exists positive constant $l_{i}$ such that

$$
\left|f_{i}(u)-f_{i}(v)\right| \leq l_{i}|u-v| \quad \forall u, v \in \mathbb{R}, i \in S \text {. }
$$

$\left(\mathrm{H}_{2}\right) \sigma_{i j}:[0, \infty) \rightarrow[0, \infty)$ is a real-valued nonnegative continuous function that satisfies

$$
\int_{0}^{\infty} \sigma_{i j}(s) d s=1, \quad i, j \in S
$$

Theorem 3.1 Under assumptions $\left(\mathrm{H}_{1}\right)$ and $\left(\mathrm{H}_{2}\right)$, system (2.3) has one unique equilibrium point if

$$
b_{i}-a_{i}-\sum_{j=1}^{n}\left(\left|p_{i j}\right|+\left|q_{i j}\right|\right) l_{j}>0 .
$$

Proof Assume that $\left(u^{* \top}, v^{* \top}\right)^{\top}$ is an equilibrium point of system (2.3), where $u^{*}=$ $\left(u_{1}^{*}, \ldots, u_{n}^{*}\right)^{\top}, v^{*}=\left(v_{1}^{*}, \ldots, v_{n}^{*}\right)^{\top}$. By assumption $\left(\mathrm{H}_{2}\right)$, we have

$$
\left\{\begin{array}{l}
a_{i} u_{i}^{*}-v_{i}^{*}=0, \\
b_{i} u_{i}^{*}-\sum_{j=1}^{n} p_{i j} f_{j}\left(u_{j}^{*}\right)-\sum_{j=1}^{n} q_{i j} f_{j}\left(u_{j}^{*}\right)-I_{i}=0 .
\end{array}\right.
$$

Denote $h(u, v)=\left(h_{1}, \ldots, h_{n}, \tilde{h}_{1}, \ldots, \tilde{h}_{n}\right)^{\top}$, where

$$
\left\{\begin{array}{l}
h_{i}=a_{i} u_{i}-v_{i}, \\
\tilde{h}_{i}=b_{i} u_{i}-\sum_{j=1}^{n}\left(p_{i j}+q_{i j}\right) f_{j}\left(u_{j}\right)-I_{i} .
\end{array}\right.
$$

Then the equilibrium point of system (2.3) is the solution of the following system:

$$
\left\{\begin{array}{l}
h_{i}=0 \\
\tilde{h}_{i}=0 .
\end{array}\right.
$$

Define a bounded open set $\Omega$ and a homotopic map $H(\lambda, u, v)$ as follows:

$$
\Omega=\left\{(u, v)^{\top}:\left|u_{i}\right| \leq \rho_{i},\left|v_{i}\right| \leq a_{i} \rho_{i}, i \in S\right\},
$$

and

$$
H(\lambda, u, v)=\lambda h(u, v)+(1-\lambda)\left(u^{\top}, v^{\top}\right)^{\top},
$$


where $u=\left(u_{1}, \ldots, u_{n}\right)^{\top}, v=\left(v_{1}, \ldots, v_{n}\right)^{\top}, \rho_{i}=\frac{1+\left|I_{i}\right|+\sum_{j=1}^{n}\left(\left|p_{i j}\right|+\left|q_{i j}\right|\right) f_{j}(0)}{b_{i}-a_{i}-\sum_{j=1}^{n}\left(\left|p_{j i}\right|+\left|q_{j i}\right|\right) l_{j}}$. By assumption $\left(\mathrm{H}_{1}\right)$, we have

$$
\begin{aligned}
\|H(\lambda, u, v)\|_{1}= & \sum_{i=1}^{n}\left|\lambda h_{i}+(1-\lambda) u_{i}\right|+\sum_{i=1}^{n}\left|\lambda \tilde{h}_{i}+(1-\lambda) v_{i}\right| \\
\geq & (1-\lambda) \sum_{i=1}^{n}\left|u_{i}\right|+(2-\lambda) \sum_{i=1}^{n}\left|v_{i}\right| \\
& +\lambda \sum_{i=1}^{n}\left(b_{i}-a_{i}-\sum_{j=1}^{n}\left(\left|p_{j i}\right|+\left|q_{j i}\right|\right) l_{j}\right)\left|u_{i}\right| \\
& -\lambda \sum_{i=1}^{n}\left(\left|I_{i}\right|+\sum_{j=1}^{n}\left(\left|p_{i j}\right|+\left|q_{i j}\right|\right) f_{j}(0)\right) .
\end{aligned}
$$

When $\left(u^{\top}, v^{\top}\right)^{\top} \in \partial \Omega$, by condition (3.1), we have

$$
\|H(\lambda, u, v)\|_{1}>0
$$

Hence, we get

$$
H(\lambda, u, v) \neq 0 \quad \text { for }\left(u^{\top}, v^{\top}\right)^{\top} \in \partial \Omega, \lambda \in[0,1]
$$

From Lemma 1.1 and 1.2, we have $\operatorname{deg}(h, \Omega, 0)=\operatorname{deg}(H, \Omega, 0)=1$ and system (2.3) has at least one solution in $\Omega$. Next, we show that the solution of system (2.3) is unique. Assume that $\left(u^{* \top}, v^{* \top}\right)^{\top}$ and $\left(\tilde{u}^{\top}, \tilde{v}^{\top}\right)^{\top}$ are two solutions of system $(2.3)$, where $u^{*}=\left(u_{1}^{*}, \ldots, u_{n}^{*}\right)^{\top}$, $v^{*}=\left(v_{1}^{*}, \ldots, v_{n}^{*}\right)^{\top}, \tilde{u}=\left(\tilde{u}_{1}, \ldots, \tilde{u}_{n}\right)^{\top}, \tilde{v}=\left(\tilde{v}_{1}, \ldots, \tilde{v}_{n}\right)^{\top}$. Thus,

$$
\left\{\begin{array}{l}
a_{i}\left(u_{i}^{*}-\tilde{u}_{i}\right)-\left(v_{i}^{*}-\tilde{v}_{i}\right)=0, \\
\left.b_{i}\left(u_{i}^{*}-\tilde{u}_{i}\right)\right)-\sum_{j=1}^{n}\left(p_{i j}+q_{i j}\right)\left(f_{j}\left(u_{j}^{*}\right)-f_{j}\left(\tilde{u}_{j}\right)\right)=0 .
\end{array}\right.
$$

From the second equation of system (3.3) and assumption $\left(\mathrm{H}_{2}\right)$, we have

$$
b_{i}\left|u_{i}^{*}-\tilde{u}_{i}\right|-\sum_{j=1}^{n}\left(\left|p_{i j}\right|+\left|q_{i j}\right|\right) l_{j}\left|u_{j}^{*}-\tilde{u}_{j}\right| \leq 0
$$

and $u^{* \top}=\tilde{u}^{\top}, v^{* \top}=\tilde{v}^{\top}$. Thus the equilibrium point of system (2.3) is unique.

The second equation of system (2.3) does not contain the term $v_{i}$ which is not a matching system. For obtaining exponential stability of system (2.3), we consider the following generalized system:

$$
\left\{\begin{aligned}
\frac{\partial u_{i}(t, x)}{\partial t}= & \sum_{k=1}^{l} \frac{\partial}{\partial x_{k}}\left(D_{i k} \frac{\partial u_{i}}{\partial x_{k}}\right)-a_{i} u_{i}+v_{i}(t, x), \\
\frac{\partial v_{i}(t, x)}{\partial t}= & \sum_{k=1}^{l} \frac{\partial}{\partial x_{k}}\left(D_{i k} \frac{\partial v_{i}}{\partial x_{k}}\right)-c_{i} v_{i}-b_{i} u_{i}+\sum_{j=1}^{n} p_{i j} f_{j}\left(u_{j}\right) \\
& +\sum_{j=1}^{n} q_{i j} \int_{-\infty}^{t} \sigma_{i j}(t-s) f_{j}\left(u_{j}(s, x)\right) d s+I_{i},
\end{aligned}\right.
$$

where $c_{i}>0, i \in S$, whose boundary conditions and initial conditions are (2.4) and (2.5), respectively. 
Definition 3.1 If $\left(u^{* \top}, v^{* \top}\right)^{\top}$ is an equilibrium point of (3.4) and $\left(u^{\top}, v^{\top}\right)^{\top}$ is any solution of (3.4) satisfying

$$
\lim _{t \rightarrow+\infty}\left[\sum_{i=1}^{n}\left\|u_{i}(t)-u_{i}^{*}(t)\right\|_{2}+\sum_{i=1}^{n}\left\|v_{i}(t)-v_{i}^{*}(t)\right\|_{2}\right] \leq M\left(\left\|\phi-u^{*}\right\|_{2}+\left\|\psi-v^{*}\right\|_{2}\right) e^{-\delta t}
$$

where $M \geq 1, \delta>0$,

$$
\left\|\phi-u^{*}\right\|_{2}=\sup _{s \in(-\infty, 0]} \sum_{i=1}^{n}\left\|u_{i}(s, x)-u_{i}^{*}\right\|_{2},\left\|\psi-v^{*}\right\|_{2}=\sup _{s \in(-\infty, 0]} \sum_{i=1}^{n}\left\|v_{i}(s, x)-v_{i}^{*}\right\|_{2} .
$$

We call $\left(u^{* \top}, v^{* \top}\right)^{\top}$ globally exponentially stable.

Theorem 3.2 Assume that system (3.4) has a unique equilibrium point. Assume further

$\left(\mathrm{H}_{3}\right)$

$$
b_{i}-a_{i}-\sum_{j=1}^{n}\left(\left|p_{i j}\right|+\left|q_{i j}\right|\right) l_{j}>0 \quad \text { and } \quad c_{i}>1 \quad \text { for } i, j \in S .
$$

Then the unique equilibrium point of system (3.4) is globally asymptotically stable.

Proof Suppose that system (3.4) has the unique equilibrium point $\left(u^{* \top}, v^{* \top}\right)^{\top}$, and $\left(u^{\top}, v^{\top}\right)^{\top}$ is any solution of system (3.4). Rewrite system (3.4) as the following form:

$$
\left\{\begin{array}{l}
\frac{\partial\left(u_{i}-u_{i}^{*}\right)}{\partial t}=\sum_{k=1}^{l} \frac{\partial}{\partial x_{k}}\left(D_{i k} \frac{\partial\left(u_{i}-u_{i}^{*}\right)}{\partial x_{k}}\right)-a_{i}\left(u_{i}-u_{i}^{*}\right)+\left(v_{i}-v_{i}^{*}\right), \\
=\sum_{k=1}^{l} \frac{\partial}{\partial x_{k}}\left(D_{i k} \frac{\partial\left(v_{i}-v_{i}^{*}\right)}{\partial x_{k}}\right)-c_{i}\left(v_{i}-v_{i}^{*}\right)-b_{i}\left(u_{i}-u_{i}^{*}\right)+\sum_{j=1}^{n} p_{i j}\left(f_{j}\left(u_{j}\right)-f_{j}\left(u_{j}^{*}\right)\right) \\
\quad+\sum_{j=1}^{n} q_{i j} \int_{-\infty}^{t} \sigma_{i j}(t-s)\left(f_{j}\left(u_{j}(s, x)\right)-f_{j}\left(u_{j}^{*}\right)\right) d s .
\end{array}\right.
$$

Multiply both sides of the first equation of (3.5) by $u_{i}-u_{i}^{*}$ and integrate them

$$
\begin{aligned}
\frac{1}{2} \frac{d}{d t} \int_{\Omega}\left(u_{i}-u_{i}^{*}\right)^{2} d x= & \sum_{k=1}^{l} \int_{\Omega}\left(u_{i}-u_{i}^{*}\right) \frac{\partial}{\partial x_{k}}\left(D_{i k} \frac{\partial\left(u_{i}-u_{i}^{*}\right)}{\partial x_{k}}\right) \\
& -\int_{\Omega} a_{i}\left(u_{i}-u_{i}^{*}\right)^{2} d x+\int_{\Omega}\left(v_{i}-v_{i}^{*}\right)\left(u_{i}-u_{i}^{*}\right) d x .
\end{aligned}
$$

Use the boundary condition (2.4), then

$$
\sum_{k=1}^{l} \int_{\Omega}\left(u_{i}-u_{i}^{*}\right) \frac{\partial}{\partial x_{k}}\left(D_{i k} \frac{\partial\left(u_{i}-u_{i}^{*}\right)}{\partial x_{k}}\right)=-\sum_{k=1}^{l} \int_{\Omega} D_{i k}\left(\frac{\partial\left(u_{i}-u_{i}^{*}\right)}{\partial x_{k}}\right)^{2} d x
$$

By (3.6) and (3.7), we have

$$
\frac{d\left\|u_{i}-u_{i}^{*}\right\|_{2}^{2}}{d t} \leq-2 a_{i}\left\|u_{i}-u_{i}^{*}\right\|_{2}^{2}+2\left\|u_{i}-u_{i}^{*}\right\|_{2}\left\|v_{i}-v_{i}^{*}\right\|_{2}
$$


i.e.

$$
\frac{d\left\|u_{i}-u_{i}^{*}\right\|_{2}}{d t} \leq-a_{i}\left\|u_{i}-u_{i}^{*}\right\|_{2}+\left\|v_{i}-v_{i}^{*}\right\|_{2} .
$$

Multiply both sides of the second equation of (3.5) by $v_{i}-v_{i}^{*}$ and integrate them

$$
\begin{aligned}
& \frac{1}{2} \frac{d}{d t} \int_{\Omega}\left(v_{i}-v_{i}^{*}\right)^{2} d x \\
& =\sum_{k=1}^{l} \int_{\Omega}\left(v_{i}-v_{i}^{*}\right) \frac{\partial}{\partial x_{k}}\left(D_{i k} \frac{\partial\left(v_{i}-v_{i}^{*}\right)}{\partial x_{k}}\right)-\int_{\Omega} c_{i}\left(v_{i}-v_{i}^{*}\right)^{2} d x \\
& \quad-\int_{\Omega} b_{i}\left(u_{i}-u_{i}^{*}\right)\left(v_{i}-v_{i}^{*}\right) d x+\sum_{j=1}^{n} p_{i j} \int_{\Omega}\left(f_{j}\left(u_{j}\right)-f_{j}\left(u_{j}^{*}\right)\right)\left(v_{i}-v_{i}^{*}\right) d x \\
& \quad+\int_{\Omega}\left(v_{i}-v_{i}^{*}\right)\left[\sum_{j=1}^{n} q_{i j} \int_{-\infty}^{t} \sigma_{i j}(t-s)\left(f_{j}\left(u_{j}(s, x)\right)-f_{j}\left(u_{j}^{*}\right)\right) d s\right] d x .
\end{aligned}
$$

Use the boundary condition (2.4), then

$$
\sum_{k=1}^{l} \int_{\Omega}\left(v_{i}-v_{i}^{*}\right) \frac{\partial}{\partial x_{k}}\left(D_{i k} \frac{\partial\left(v_{i}-v_{i}^{*}\right)}{\partial x_{k}}\right)=-\sum_{k=1}^{l} \int_{\Omega} D_{i k}\left(\frac{\partial\left(v_{i}-v_{i}^{*}\right)}{\partial x_{k}}\right)^{2} d x
$$

In view of (3.9), (3.10), assumption $\left(\mathrm{H}_{1}\right)$, and Hölder's inequality, we have

$$
\begin{aligned}
\frac{d\left\|v_{i}-v_{i}^{*}\right\|_{2}^{2}}{d t} \leq & -2 c_{i}\left\|v_{i}-v_{i}^{*}\right\|_{2}^{2}+2 b_{i}\left\|u_{i}-u_{i}^{*}\right\|_{2}\left\|v_{i}-v_{i}^{*}\right\|_{2} \\
& +2 \sum_{j=1}^{n}\left|p_{i j}\right| l_{j}\left\|u_{j}-u_{j}^{*}\right\|_{2}\left\|v_{i}-v_{i}^{*}\right\|_{2} \\
& +2 \sum_{j=1}^{n}\left|q_{i j}\right| l_{j} \int_{-\infty}^{t} \sigma_{i j}(t-s)\left\|v_{i}-v_{i}^{*}\right\|_{2}\left\|u_{j}-u_{j}^{*}\right\|_{2} d s
\end{aligned}
$$

i.e.

$$
\begin{aligned}
\frac{d\left\|v_{i}-v_{i}^{*}\right\|_{2}}{d t} \leq & -c_{i}\left\|v_{i}-v_{i}^{*}\right\|_{2}+b_{i}\left\|u_{i}-u_{i}^{*}\right\|_{2}+\sum_{j=1}^{n}\left|p_{i j}\right| l_{j}\left\|u_{j}-u_{j}^{*}\right\|_{2} \\
& +\sum_{j=1}^{n}\left|q_{i j}\right| l j \int_{-\infty}^{t} \sigma_{i j}(t-s)\left\|u_{j}-u_{j}^{*}\right\|_{2} d s .
\end{aligned}
$$

Let

$$
\left\{\begin{array}{l}
\phi_{i}\left(\xi_{i}\right)=b_{i}-a_{i}-\xi_{i}-l_{j} \sum_{j=1}^{n}\left(\left|p_{i j}\right|+\left|q_{i j}\right| \int_{0}^{\infty} e^{\xi_{i} s} \sigma_{i j}(s) d s\right), \quad \xi_{i} \in[0, \infty), \\
\psi_{i}\left(\eta_{i}\right)=c_{i}-1-\eta_{i}, \quad \eta_{i} \in[0, \infty),
\end{array}\right.
$$

where $i \in S$. By assumption $\left(\mathrm{H}_{3}\right)$, we have $\psi_{i}(0)=c_{i}-1>0$ for $i \in S$. By condition (3.1), $\phi_{i}(0)=b_{i}-a_{i}-\sum_{j=1}^{n}\left(\left|p_{i j}\right|+\left|q_{i j}\right|\right) l_{j}>0$ for $i \in S$. It is easy to see that $\phi_{i}\left(\xi_{i}\right)$ and $\psi_{i}\left(\eta_{i}\right)$ are 
continuous for $\xi_{i}, \eta_{i} \in[0, \infty)$, and $\phi_{i}^{\prime}\left(\xi_{i}\right)<0$ and $\psi_{i}^{\prime}\left(\eta_{i}\right)<0$ i.e. $\phi_{i}\left(\xi_{i}\right)$ and $\psi_{i}\left(\eta_{i}\right)$ are strictly decreasing functions on $[0, \infty)$. Hence, there exist constants $\xi_{i}^{*}, \eta_{i}^{*} \in(0, \infty)$ such that

$$
\left\{\begin{array}{l}
\phi_{i}\left(\xi_{i}^{*}\right)=b_{i}-a_{i}-\xi_{i}^{*}-l_{j} \sum_{j=1}^{n}\left(\left|p_{i j}\right|+\left|q_{i j}\right| \int_{0}^{\infty} e^{\xi_{i}^{*} s} \sigma_{i j}(s) d s\right)=0, \\
\psi_{i}\left(\eta_{i}^{*}\right)=c_{i}-1-\eta_{i}^{*}=0 .
\end{array}\right.
$$

Choose $\delta=\min _{i \in S}\left\{\xi_{i}^{*}, \eta_{i}^{*}\right\}>0$. By (3.13) we get

$$
\left\{\begin{array}{l}
\phi_{i}(\delta)=b_{i}-a_{i}-\delta-l_{j} \sum_{j=1}^{n}\left(\left|p_{i j}\right|+\left|q_{i j}\right| \int_{0}^{\infty} e^{\xi_{i}^{*} s} \sigma_{i j}(s) d s\right) \geq 0 \\
\psi_{i}(\delta)=c_{i}-1-\delta \geq 0
\end{array}\right.
$$

Construct the following Lyapunov functional:

$$
\begin{aligned}
V(t)= & \sum_{i=1}^{n}\left(e^{\delta t}\left\|u_{i}-u_{i}^{*}\right\|_{2}+e^{\delta t}\left\|v_{i}-v_{i}^{*}\right\|_{2}\right. \\
& \left.+\sum_{j=1}^{n}\left|q_{i j}\right| l j \int_{0}^{\infty} \sigma_{i j}(s) \int_{t-s}^{t}\left\|u_{j}(\rho, x)-u_{j}^{*}\right\|_{2} e^{\delta(\rho+s)} d \rho d s\right) .
\end{aligned}
$$

Calculating the upper right Dini derivative of $V(t)$ along the solutions of system (3.4), in view of assumption $\left(\mathrm{H}_{3}\right)$, we have

$$
\begin{aligned}
D^{+} V(t) \leq & e^{\delta t} \sum_{i=1}^{n}\left(\left(\delta+b_{i}-a_{i}\right)\left\|u_{i}-u_{i}^{*}\right\|_{2}+(\delta+1)\left\|v_{i}-v_{i}^{*}\right\|_{2}\right. \\
& \left.+\sum_{j=1}^{n}\left|p_{i j}\right| l_{j}\left\|u_{j}-u_{j}^{*}\right\|_{2}+\sum_{j=1}^{n}\left|q_{i j}\right| l_{j} \int_{-\infty}^{t} \sigma_{i j}(t-s)\left\|u_{j}-u_{j}^{*}\right\|_{2} d s\right) \\
= & e^{\delta t} \sum_{j=1}^{n}\left[\delta+b_{i}-a_{i}+\sum_{i=1}^{n}\left|p_{i j}\right| l_{j}+\sum_{i=1}^{n}\left|q_{i j}\right| l j \int_{-\infty}^{t} \sigma_{i j}(t-s) d s\right] \\
& \times\left\|u_{j}-u_{j}^{*}\right\|_{2} \\
& +e^{\delta t} \sum_{i=1}^{n}\left(\delta+1-c_{i}\right)\left\|v_{i}-v_{i}^{*}\right\|_{2} \\
= & -e^{\delta t} \sum_{j=1}^{n}\left[a_{i}-\delta-b_{i}-\sum_{i=1}^{n}\left|p_{i j}\right| l_{j}-\sum_{i=1}^{n}\left|q_{i j}\right| l j \int_{-\infty}^{t} \sigma_{i j}(t-s) d s\right] \\
& \times\left\|u_{j}-u_{j}^{*}\right\|_{2} \\
& -e^{\delta t} \sum_{i=1}^{n}\left(c_{i}-\delta-1\right)\left\|v_{i}-v_{i}^{*}\right\|_{2} \\
\leq & 0 .
\end{aligned}
$$

By (3.16) we have

$$
V(t) \leq V(0) \quad \text { for } t>0 \text {. }
$$


By (3.15) we have

$$
V(t) \geq e^{\delta t} \sum_{i=1}^{n}\left(\left\|u_{i}-u_{i}^{*}\right\|_{2}+\left\|v_{i}-v_{i}^{*}\right\|_{2}\right)
$$

and

$$
\begin{aligned}
V(0)= & \sum_{i=1}^{n}\left(\left\|u_{i}(0, x)-u_{i}^{*}\right\|_{2}+\left\|v_{i}(0, x)-v_{i}^{*}\right\|_{2}\right. \\
& \left.+\sum_{j=1}^{n}\left|q_{i j}\right| l j \int_{0}^{\infty} \sigma_{i j}(s) \int_{-s}^{0}\left\|u_{j}(\rho, x)-u_{j}^{*}\right\|_{2} e^{\delta(\rho+s)} d \rho d s\right) \\
\leq & \left(1+\sum_{j=1}^{n}\left|q_{j i}\right| l_{i} \int_{0}^{\infty} \sigma_{j i}(s) \int_{-s}^{0} e^{\delta(\rho+s)} d \rho d s\right) \sup _{s \in[0, \infty)} \sum_{i=1}^{n}\left\|u_{i}(s, x)-u_{i}^{*}\right\|_{2} \\
& +\sup _{s \in[0, \infty)} \sum_{i=1}^{n}\left\|v_{i}(s, x)-v_{i}^{*}\right\|_{2} \\
\leq & M\left(\sup _{s \in[0, \infty)} \sum_{i=1}^{n}\left\|u_{i}(s, x)-u_{i}^{*}\right\|_{2}+\sup _{s \in[0, \infty)} \sum_{i=1}^{n}\left\|v_{i}(s, x)-v_{i}^{*}\right\|_{2}\right),
\end{aligned}
$$

where

$$
M=\max _{i \in S}\left\{\sum_{j=1}^{n}\left|q_{j i}\right| l_{i} \int_{0}^{\infty} \sigma_{j i}(s) \int_{-s}^{0} e^{\delta(\rho+s)} d \rho d s, 1\right\} .
$$

From (3.17)-(3.19), we have

$$
\begin{aligned}
& \sum_{i=1}^{n}\left\|u_{i}(s, x)-u_{i}^{*}\right\|_{2}+\sum_{i=1}^{n}\left\|v_{i}(s, x)-v_{i}^{*}\right\|_{2} \\
& \quad \leq M e^{-\delta t}\left(\sup _{s \in[0, \infty)} \sum_{i=1}^{n}\left\|u_{i}(s, x)-u_{i}^{*}\right\|_{2}+\sup _{s \in[0, \infty)} \sum_{i=1}^{n}\left\|v_{i}(s, x)-v_{i}^{*}\right\|_{2}\right) \\
& \quad \leq M\left(\left\|\phi-u^{*}\right\|_{2}+\left\|\psi-v^{*}\right\|_{2}\right) e^{-\delta t} .
\end{aligned}
$$

The proof is completed.

Remark 3.1 We really want to obtain global exponential stability for system (2.3). However, system (2.3) has strong non match, which is different from some reaction-diffusion systems in [11-13]. For system (2.3), constructing a proper Lyapunov functional is very difficult because of its strong non match. Hence, we study system (3.4) which is similar to system (2.3) and obtain global exponential stability of system (3.4) by constructing a proper Lyapunov functional and some mathematical analysis technique. We hope that some authors will solve the global exponential stability problem of system (2.3) by the innovative approach.

Remark 3.2 In this paper, Lemma 1.1 and Lemma 1.2 belong to topological degree theory. We use Lemma 1.1 and Lemma 1.2 for obtaining the existence result of system (2.3). We 
give some details for the use of topological degree theory as follows: firstly, we show that the mapping $H(\lambda, x)=y$ has no solutions in $\partial \Omega$ for $\lambda \in[0,1]$ and $y \in R^{n} \backslash H(\lambda, \partial \Omega)$; after that, we prove $\operatorname{deg}(H(x), \Omega, y) \neq 0$. Then there exists at least one solution $H(x)=y$ in $\Omega$.

Remark 3.3 In Theorem 3.1, for obtaining

$$
H(\lambda, u, v) \neq 0 \quad \text { for }\left(u^{\top}, v^{\top}\right)^{\top} \in \partial \Omega, \lambda \in[0,1]
$$

by using some inequality technique, we give estimation of $\|H(\lambda, u, v)\|_{1}$. In Theorem 3.2, for obtaining the stability result of system (3.4), we construct a proper Lyapunov functional and use some complex mathematical analysis skills.

\section{Numerical example}

This section presents an example that demonstrates the validity of our theoretical results. Consider the following two-dimensional neural networks with distributed delays and reaction-diffusion terms:

$$
\left\{\begin{aligned}
\frac{\partial u_{i}(t, x)}{\partial t}= & \sum_{k=1}^{l} \frac{\partial}{\partial x_{k}}\left(D_{i k} \frac{\partial u_{i}}{\partial x_{k}}\right)-a_{i} u_{i}+v_{i}(t, x), \\
\frac{\partial v_{i}(t, x)}{\partial t}= & \sum_{k=1}^{l} \frac{\partial}{\partial x_{k}}\left(D_{i k} \frac{\partial v_{i}}{\partial x_{k}}\right)-b_{i} u_{i}+\sum_{j=1}^{n} p_{i j} f_{j}\left(u_{j}\right) \\
& +\sum_{j=1}^{n} q_{i j} \int_{-\infty}^{t} \sigma_{i j}(t-s) f_{j}\left(u_{j}(s, x)\right) d s+I_{i},
\end{aligned}\right.
$$

where

$$
\begin{aligned}
& i, j, k=1,2, \quad D_{i k}=1>0, \quad \int_{0}^{\infty} \sigma_{i j}(s) d s=\int_{0}^{\infty} e^{-s} d s=1, \\
& b_{i}=5, \quad a_{i}=2, \quad p_{i j}=q_{i j}=0.5, \quad f_{i}(y)=1+y \quad \text { for } y \in \mathbb{R} .
\end{aligned}
$$

Obviously,

$$
l_{i}=1, \quad\left|f_{i}(u)-\right| f_{i}(v)|| \leq|u-v| \quad \text { for } u, v \in \mathbb{R}
$$

and

$$
b_{i}-a_{i}-\sum_{j=1}^{n}\left(\left|p_{i j}\right|+\left|q_{i j}\right|\right) l_{j}=1>0 .
$$

Thus, all assumptions of Theorem 3.1 hold and (4.1) has a unique equilibrium point. The solution of system (4.1) is shown in Fig. 1.

Remark 4.1 For all we know, the INNs with reaction-diffusion terms and distributed delays are a new model in the present paper. Using topological degree theory and the mathematical analysis technique, we get some brand new results on the existence, uniqueness, and global exponential stability of solution of INNs. We can confirm the truth of the proposed methods, for example, in $[4-6,12,13]$ cannot be generalized to the problems studied in this article. 


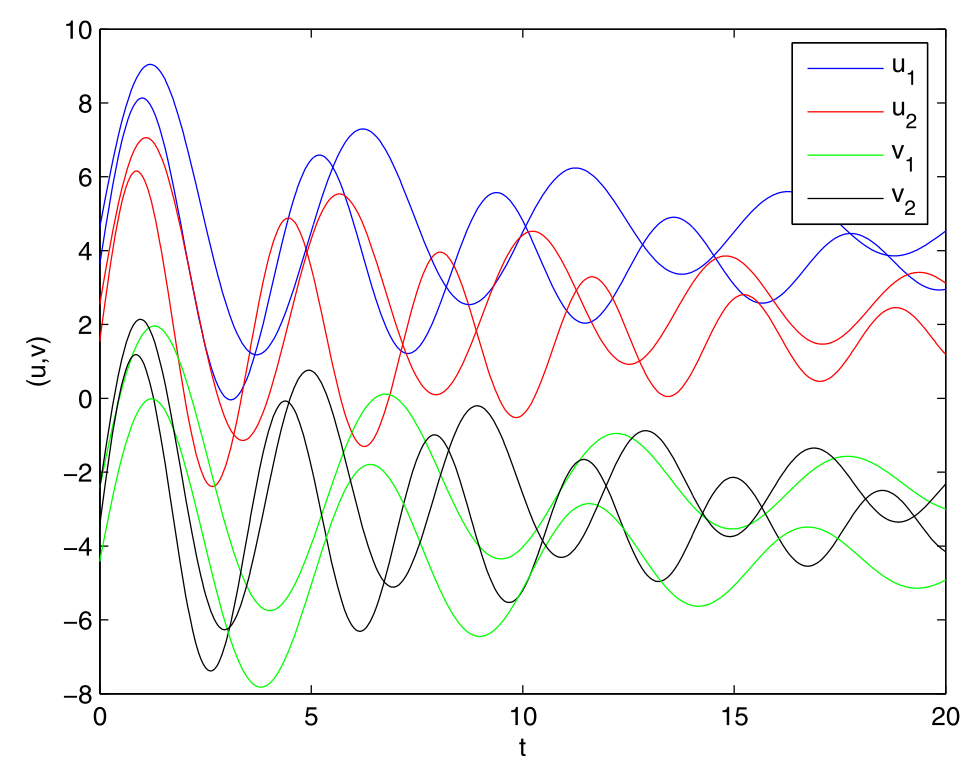

Figure 1 State trajectories of system (4.1)

Remark 4.2 In [13], the authors gave some numerical simulations of a periodic solution for a class of neural networks with reaction-diffusion terms and both variable and unbounded delays. Numerical simulations in the present paper show the solution properties of second-order system, but neural networks in [13] are a first-order system. Hence, numerical simulations in the present paper are more complicated than the existing ones. For more similar numerical simulations of a first-order system, see e.g. [10-12].

\section{Conclusions and discussions}

In this paper we study the dynamic behaviors of solutions for inertial neural networks with reaction-diffusion terms and distributed delays. First, by applying topological degree theory to the system, we get a set of sufficient conditions for guaranteeing the existence and uniqueness of solutions. Then, the global exponential stability of the equilibrium point is obtained by using the Lyapunov functional. The efficacy of the obtained results has been demonstrated by numerical simulations. It is important to note that the practical implementation of INNs is typically encountered with certain type of uncertainties such as interval parameters. These results can be applied to design globally exponentially stable networks and thus have important significance in both theory and applications. Extending the results of this paper to INNs with interval uncertainties proves to be an interesting problem.

Acknowledgements

The authors would like to thank the editor and the referees for their valuable comments and suggestions that improved the quality of our paper.

Funding

Not applicable. 
Competing interests

The author declares that they have no competing interests.

\section{Authors' contributions}

All authors contributed equally to the writing of this paper. All authors read and approved the final manuscript.

\section{Publisher's Note}

Springer Nature remains neutral with regard to jurisdictional claims in published maps and institutional affiliations.

Received: 14 July 2020 Accepted: 4 March 2021 Published online: 12 March 2021

\section{References}

1. Wheeler, D., Schieve, W.: Stability and chaos in an inertial two-neuron system. Phys. D: Nonlinear Phenom. 105, 26-284 (1997)

2. Angelaki, D.E., Correia, M.J.: Models of membrane resonance in pigeon semicircular canal type II hair cells. Biol. Cybern. 65, 1-10 (1991)

3. Yin, H., Du, B.: Stochastic patch structure Nicholson's blowflies system with mixed delays. Adv. Differ. Equ. 386, 1-11 (2020)

4. Tu, Z., Cao, J., Hayat, T.: Global exponential stability in Lagrange sense for inertial neural networks with time-varying delays. Neurocomputing 171, 524-531 (2016)

5. Wang, J., Tian, L.: Global Lagrange stability for inertial neural networks with mixed time-varying delays. Neurocomputing 235, 140-146 (2017)

6. Yin, H., Du, B., Yang, Q., Duan, F.: Existence of homoclinic orbits for a singular differential equation involving p-Laplacian. J. Funct. Spaces 5, 1-7 (2020)

7. Tu, Z., Cao, J., Hayat, T.: Matrix measure based dissipativity analysis for inertial delayed uncertain neural networks. Neural Netw. 75, 47-55 (2016)

8. Wan, P., Jian, J.: Global convergence analysis of impulsive inertial neural networks with time-varying delays. Neurocomputing 245, 68-76 (2017)

9. Draye, J., Winters, J., Cheron, G.: Self-selected modular recurrent neural networks with postural and inertial subnetworks applied to complex movements. Biol. Cybern. 87, 27-39 (2002)

10. He, Q., Kang, L.: Existence and stability of global solution for generalized Hopfield neural network system. Neural Parallel Sci. Comput. 2, 165-175 (1994)

11. Li, X., Cao, J.: Delay-independent exponential stability of stochastic Cohen-Grossberg neural networks with time-varying delays and reaction-diffusion terms. Nonlinear Dyn. 50, 363-371 (2007)

12. Carpenter, G.A.: A geometric approach to singular perturbation problems with application to nerve impulse equations. J. Differ. Equ. 23, 355-367 (1977)

13. Zhao, Z., Song, Q., Zhang, J.: Exponential periodicity and stability of neural networks with reaction-diffusion terms and both variable and unbounded delays. Comput. Math. Appl. 51, 475-486 (2006)

14. Deimling, K.: Nonlinear Functional Analysis. Springer, Berlin (1985)

\section{Submit your manuscript to a SpringerOpen ${ }^{\circ}$ journal and benefit from:}

- Convenient online submission

- Rigorous peer review

- Open access: articles freely available online

- High visibility within the field

- Retaining the copyright to your article

Submit your next manuscript at $\gg$ springeropen.com 